\title{
Diagnóstico histopatológico das lesões bucais. A experiência do serviço de cirurgia e traumatologia bucomaxilofacial do complexo Hospitalar Santa Casa de Porto Alegre
}

\author{
Histopathological diagnosis of oral lesions. The experience of \\ maxillofacial surgery service of the hospital complex \\ Santa Casa de Porto Alegre
}

Bruno Trevisan*

João Carlos Birnfeld Wagner ${ }^{* *}$

Maurício Roth Volkweis ${ }^{* * *}$

\section{Resumo}

Objetivo: este estudo avaliou a prevalência de lesões bucais em diagnósticos por meio de exame histopatológico, atendidas pelo serviço de cirurgia e traumatologia bucomaxilofacial de um hospital na região Sul do Brasil, durante o período de janeiro de 2000 a março de 2012. Métodos: os dados foram obtidos pelos laudos emitidos pelo laboratório de patologia. Foram analisados 707 laudos histopatológicos. Resultados: os resultados demonstraram que as lesões inflamatórias foram as mais frequentes $(21,4 \%)$. Os diagnósticos acometeram mais frequentemente mulheres $(60,3 \%)$ e, quanto à faixa etária, pacientes de até 30 anos de idade (40,4\%). O folículo pericoronário $(15,4 \%)$ foi a lesão mais frequente e fortemente associada com pacientes de até 30 anos independentemente do sexo, assim como as patologias de glândulas salivares. Conclusões: as lesões inflamatórias estão mais presentes em mulheres com mais de 50 anos e em homens com mais de 60. As neoplasias malignas também foram mais presentes nos homens com mais de 60 anos de idade.

Palavras-chave: Epidemiologia. Diagnóstico.

\section{Introdução}

O processo de diagnóstico é um desafio constante na vida dos profissionais que atuam na área de saúde. Independentemente da região anatômica, o exame clínico, muitas vezes, não é suficiente para se obter um diagnóstico preciso ${ }^{1}$. Para o auxílio do profissional, há, atualmente, inúmeros exames complementares. O exame histopatológico é uma importante ferramenta para o cirurgião, pois, juntamente com os dados clínicos, o auxilia a decidir a sua conduta para o tratamento de seus pacientes ${ }^{2}$.

Muitas patologias têm maior prevalência em um determinado grupo da população. Essa distribuição pode variar de acordo com algumas variáveis do indivíduo, como suas características físicas e étnicas ou em relação a seus hábitos e sua história de vida $^{3}$. O conhecimento dessa relação perfil-paciente com determinada patologia pode auxiliar o cirurgião bucomaxilofacial no seu diagnóstico ${ }^{4}$.

Cirurgião-dentista residente em CTBMF da Santa Casa de Porto Alegre, RS, Brasil.

*** Especialista, mestre e doutor em Cirurgia e Traumatologia Bucomaxilofacial, Serviço de CTBMF da Santa Casa de Porto Alegre, RS, Brasil. Doutor em Estomatologia, Especialista e Mestre em Cirurgia e Traumatologia Bucomaxilofacial Serviço de CTBMF da Santa Casa de Porto Alegre, RS, Brasil. 
Estudos que analisam a forma como os perfis dos pacientes os predispõem à ocorrência de um tipo de lesão são realizados em todo o mundo. No entanto, fatores socioeconômicos, étnicos, geográficos, entre outras características, podem influenciar na prevalência e na distribuição das patologias ${ }^{5-7}$.

Muitos estudos têm buscado definir a relação entre determinadas lesões e a sua população adscrita a partir de dados e análise de grupos populacionais. Alguns utilizam prontuários, como estudos realizados em instituições hospitalares e centros de referência ${ }^{8,9}$, enquanto outros utilizam dados e fichas, bem como exames provenientes de bióp$\operatorname{sias}^{4-7,10-12}$.

O Brasil possui dimensões continentais e uma população com enorme miscigenação étnica. Por esse motivo, estudos epidemiológicos e levantamentos sobre a ocorrência e a caracterização das patologias que acometem as populações fazem-se necessários.

\section{Métodos}

Esta pesquisa tem como base metodológica um estudo retrospectivo, a partir dos resultados obtidos pelo laboratório de patologia, referentes a pacientes submetidos à biópsia atendidos pelo Serviço de Cirurgia e Traumatologia Bucomaxilofacial do Complexo Hospitalar Santa Casa de Misericórdia de Porto Alegre. Ao todo, foram emitidos 707 laudos histopatológicos, do período entre janeiro de 2000 a março de 2012.

Os laudos dos exames anatomopatológicos foram acessados nos computadores da instituição ligados em rede. Os pesquisadores, membros do corpo clínico e com livre acesso a esses laudos pelo sistema do laboratório de patologia, foram os responsáveis pela coleta de dados, obtidos através do nome de cada profissional que atuou na equipe durante o período. O sistema mantém os laudos emitidos associados ao nome e ao número de registro profissional do cirurgião responsável pela biópsia.

As lesões bucais foram classificadas em dez categorias: de origem inflamatória, alterações epiteliais, neoplasias benignas, neoplasias malignas, cistos odontogênicos, tumores odontogênicos, patologias ósseas, patologias de glândulas salivares, estruturas anatômicas e outras. Foram analisadas, também, as variáveis idade e sexo.

Os dados foram submetidos à análise estatística, sendo usado o coeficiente de contingência, qui-quadrado e análise de correspondência (Anacor). Todas as análises foram feitas com o pacote SPSS® 20.0. O projeto foi aprovado junto ao Comitê de Ética do Complexo Hospitalar Santa Casa de Misericórdia de Porto Alegre, sob parecer no 77380.

\section{Resultados}

Durante o período de janeiro de 2000 a março de 2012, foram emitidos 707 laudos histopatológicos de lesões submetidas à biópsia ou exérese pelo serviço. Destes indivíduos, 426 (60,3\%) eram do sexo feminino e 281 (39,7\%), do sexo masculino. A Tabela 1 mostra os resultados dos diagnósticos de acordo com a categoria de cada lesão, onde a categoria mais frequente foi a das lesões de origem inflamatória $(21,4 \%)$, seguida por estruturas anatômicas $(18,1 \%)$ e cistos odontogênicos $(13,6 \%)$. Foram diagnosticadas 36 neoplasias malignas, representando $5,1 \%$ da amostra, sendo o carcinoma epidermoide o diagnóstico mais frequente.

Tabela 1 - Ocorrência de lesões bucais de acordo com as categorias de diagnóstico

\begin{tabular}{l|c|c}
\hline \multicolumn{1}{c|}{ Categoria da lesão } & N total & $\%$ \\
\hline Estruturas anatômicas & 128 & 18,1 \\
Lesões inflamatórias & 151 & 21,4 \\
Alterações epiteliais & 31 & 4,4 \\
Cistos odontogênicos & 96 & 13,6 \\
Tumores odontogênicos & 32 & 4,5 \\
Patologias ósseas & 71 & 10,0 \\
Patologias de glândula salivar & 52 & 7,4 \\
Neoplasias benignas & 84 & 11,9 \\
Neoplasias malignas & 36 & 5,1 \\
Outros & 26 & 3,7 \\
Total & 707 & 100 \\
\hline
\end{tabular}

$p=0,05$

As lesões mais comuns entre todos os diagnósticos foram: o folículo pericoronário, com 109 casos $(15,4 \%)$, seguido da hiperplasia papilar inflamatória, com 89 casos $(12,5 \%)$, e do cisto radicular, com 63 casos $(8,9 \%)$. A Tabela 2 demonstra os diagnósticos mais comuns em cada categoria.

A idade dos indivíduos da amostra variou entre 1 e 103 anos de idade, com média de 39 anos. A Tabela 3 demonstra a separação pela idade categorizada. As faixas etárias mais frequentes foram dos pacientes de até 20 anos $(20,2 \%)$, de 21 a 30 anos $(20,2 \%)$ e com mais de 60 anos de idade $(16,5 \%)$. As Tabelas 4 e 5 demonstram as variáveis do sexo e da idade categorizada, individualmente, com as categorias das lesões. Quando se analisam as medidas de associação - qui-quadrado e/ou coeficiente de contingência - entre as variáveis nominais independentes sexo e idade categorizada com a variável nominal dependente tipo de lesão, constata-se o fato de existir alguma relação entre as duas variáveis independentes e a variável dependente. 
Tabela 2 - Diagnóstico mais comum (por categoria) de acordo com laudos histopatológicos

\begin{tabular}{|c|c|c|c|c|c|c|c|c|}
\hline & \multirow[b]{2}{*}{ Idade } & \multirow{2}{*}{\multicolumn{3}{|c|}{$\mathrm{N}$ total }} & \multirow{3}{*}{$\begin{array}{c}\% \\
20,2\end{array}$} \\
\hline & & & & & & & & \\
\hline Diagnósticos & $\mathrm{N}$ & $\%$ Cat & total & Até 20 anos & \multicolumn{3}{|c|}{143} & \\
\hline Estruturas anatômicas & & & 128 & De 21 a 30 anos & \multicolumn{3}{|c|}{143} & 20,2 \\
\hline Folículo pericoronário & 109 & 85 & & De 31 a 40 anos & \multicolumn{3}{|c|}{92} & 13,0 \\
\hline Lesões inflamatórias & & & 151 & De 41 a 50 anos & \multicolumn{3}{|c|}{113} & 16,0 \\
\hline Hiperplasia papilar inflamatória & 89 & 58,9 & & De 51 a 60 anos & \multicolumn{3}{|c|}{99} & 14,0 \\
\hline Tecido conjuntivo com infla & & & & Mais de 60 anos & \multicolumn{3}{|c|}{117} & 16,5 \\
\hline crônica & 30 & 19,9 & & Total & \multicolumn{3}{|c|}{707} & 100 \\
\hline Alterações epiteliais & & & 31 & & & & & \\
\hline Hiperparaceratose e acantose & 15 & 48,4 & & \multicolumn{5}{|c|}{$\begin{array}{l}\text { Tabela } 4 \text { - Ocorrência das lesões (por categoria) de acordo com } \\
\text { sexo }(p=0,05)\end{array}$} \\
\hline Mucosa escamosa com acantose & 13 & 41,9 & \multirow[b]{2}{*}{32} & Categoria da lesão & & Masc. & Fem. & Total \\
\hline $\begin{array}{l}\text { Tumores odontogênicos } \\
\text { Tumor odontogênico ceratocístico }\end{array}$ & 11 & 34,4 & & Estruturas anatômicas & $\begin{array}{l}\mathrm{N} \\
\%\end{array}$ & \begin{tabular}{c|c}
37 \\
13,2
\end{tabular} & $\begin{array}{c}91 \\
21.4\end{array}$ & $\begin{array}{r}128 \\
18,1\end{array}$ \\
\hline Odontoma & 11 & 34,4 & & \multirow{2}{*}{ Lesões inflamatórias } & $\mathrm{N}$ & 49 & 102 & 151 \\
\hline Ameloblastoma & 7 & 21,9 & & & $\%$ & 17,4 & 23,9 & 21,4 \\
\hline Cistos odontogênicos & & & 96 & \multirow[t]{2}{*}{ Alterações epiteliais } & $\mathrm{N}$ & 9 & 22 & 31 \\
\hline Cisto radicular & 63 & 65,6 & & & $\%$ & 3,2 & 5,2 & 4,4 \\
\hline Cisto dentígero & 24 & 25,0 & & \multirow[t]{2}{*}{ Cistos odontogênicos } & $\mathrm{N}$ & 55 & 41 & 96 \\
\hline Patologias ósseas & & & \multirow{2}{*}{71} & & $\%$ & 19,6 & 9,6 & 13,6 \\
\hline Granuloma periférico de células & & & & \multirow{2}{*}{ Tumores odontogênicos } & $\mathrm{N}$ & 10 & 22 & 32 \\
\hline gigantes & 8 & 11,3 & & & $\%$ & 3,6 & 5,2 & 4,5 \\
\hline Displasia fibrosa & 7 & 10,0 & & Patologias ósseas & $\mathrm{N}$ & 27 & 44 & 71 \\
\hline Patologias de glândula salivar & & & 52 & \multirow{5}{*}{$\begin{array}{l}\text { Patologias de glândula } \\
\text { salivar } \\
\text { Neoplasias benignas }\end{array}$} & $\%$ & 9,6 & 10,3 & 10 \\
\hline Mucocele & 33 & 63,5 & & & $\mathrm{~N}$ & 25 & 27 & 52 \\
\hline Neoplasias benignas & & & 84 & & $\%$ & 8,9 & 6,3 & 7,4 \\
\hline Fibroma & 19 & 22,6 & & & $\mathrm{~N}$ & 34 & 50 & 84 \\
\hline Papiloma & 19 & 22,6 & & & $\%$ & 12,1 & 11,7 & 11,9 \\
\hline Hemangioma & 16 & 19,0 & & Neoplasias malignas & $\mathrm{N}$ & 20 & 16 & 36 \\
\hline Neoplasias malignas & & & \multirow{3}{*}{36} & \multirow{3}{*}{ Outros } & & 7,1 & 3,8 & 5,1 \\
\hline Carcinoma epidermoide & 21 & - & & & IV & 15 & 11 & 26 \\
\hline & 21 & 58,3 & & & $\%$ & 3,3 & 2,6 & 3,1 \\
\hline & & & & Total & $\begin{array}{l}\mathrm{N} \\
\%\end{array}$ & $\begin{array}{l}281 \\
39,7\end{array}$ & $\begin{array}{l}426 \\
60,3\end{array}$ & $\begin{array}{l}707 \\
100\end{array}$ \\
\hline
\end{tabular}

Tabela 5 - Ocorrência das lesões (por categoria) de acordo com a idade categorizada $(p=0,05)$

\begin{tabular}{|c|c|c|c|c|c|c|c|c|}
\hline Categoria da lesão & Idade & Até 20 & 21 a 30 & 31 a 40 & 41 a 50 & 51 a 60 & Mais de 60 & Total \\
\hline \multirow[t]{2}{*}{ Estruturas anatômicas } & $\mathrm{N}$ & 54 & 54 & 8 & 9 & 2 & 1 & 128 \\
\hline & $\%$ & 37,8 & 37,8 & 8,7 & 8,0 & 2,0 & 0,9 & 18,1 \\
\hline \multirow{2}{*}{ Lesões inflamatórias } & $\mathrm{N}$ & 11 & 15 & 18 & 34 & 30 & 43 & 151 \\
\hline & $\%$ & 7,7 & 10,5 & 19,6 & 30,1 & 30,3 & 36,8 & 21,4 \\
\hline \multirow[t]{2}{*}{ Alterações epiteliais } & $\mathrm{N}$ & 2 & 3 & 4 & 9 & 3 & 10 & 31 \\
\hline & $\%$ & 1,4 & 2,1 & 4,3 & 8,0 & 3,0 & 8,5 & 4,4 \\
\hline \multirow[t]{2}{*}{ Cistos odontogênicos } & $\mathrm{N}$ & 15 & 26 & 15 & 18 & 8 & 14 & 96 \\
\hline & $\%$ & 10,5 & 18,2 & 16,3 & 15,9 & 8,1 & 12,0 & 13,6 \\
\hline \multirow[t]{2}{*}{ Tumores odontogênicos } & $N$ & 9 & 8 & 7 & 2 & 2 & 3 & 32 \\
\hline & $\%$ & 6,3 & 5,6 & 7,6 & 1,8 & 1,8 & 2,6 & 4,5 \\
\hline \multirow[t]{2}{*}{ Patologias ósseas } & $\mathrm{N}$ & 13 & 16 & 15 & 4 & 16 & 7 & 71 \\
\hline & $\%$ & 9,1 & 11,2 & 16,3 & 3,5 & 16,2 & 6,0 & 10 \\
\hline \multirow{2}{*}{$\begin{array}{l}\text { Patologias de glândula } \\
\text { salivar }\end{array}$} & $\mathrm{N}$ & 22 & 10 & 7 & 7 & 2 & 4 & 52 \\
\hline & $\%$ & 15,4 & 7,0 & 7,6 & 6,2 & 2,0 & 3,4 & 7,4 \\
\hline \multirow[t]{2}{*}{ Neoplasias benignas } & $\mathrm{N}$ & 14 & 8 & 10 & 14 & 20 & 18 & 84 \\
\hline & $\%$ & 9,8 & 5,6 & 10,9 & 12,4 & 20,2 & 15,4 & 11,9 \\
\hline \multirow[t]{2}{*}{ Neoplasias malignas } & $\mathrm{N}$ & 0 & 2 & 2 & 10 & 10 & 12 & 36 \\
\hline & $\%$ & 0,0 & 1,4 & 2,2 & 8,8 & 10,1 & 10,3 & 5,1 \\
\hline \multirow[t]{2}{*}{ Outros } & $\mathrm{N}$ & 3 & 1 & 6 & 6 & 5 & 5 & 26 \\
\hline & $\%$ & 2,1 & 0,7 & 6,5 & 5,3 & 5,1 & 4,3 & 3,7 \\
\hline \multirow[t]{2}{*}{ Total } & $\mathrm{N}$ & 143 & 143 & 92 & 113 & 99 & 117 & 707 \\
\hline & $\%$ & 20,2 & 20,2 & 13,0 & 16,0 & 14,0 & 16,5 & 100 \\
\hline
\end{tabular}

Tabela 3 - Ocorrência de lesões de acordo com idade categorizada

Tabela 4 - Ocorrência das lesões (por categoria) de acordo com o (1) 
O coeficiente de contingência entre tipo de lesão e idade categorizada resultou em $\mathrm{C}=0,53(\mathrm{p}=$ $0,000)$ entre os homens e $\mathrm{C}=0,55(\mathrm{p}=0,000)$ entre as mulheres, o que sugere que a associação depende do sexo. Para demostrar a associação entre as variáveis, foi realizada uma Anacor entre as categorias de lesões, faixa etária e os sexos separadamen-

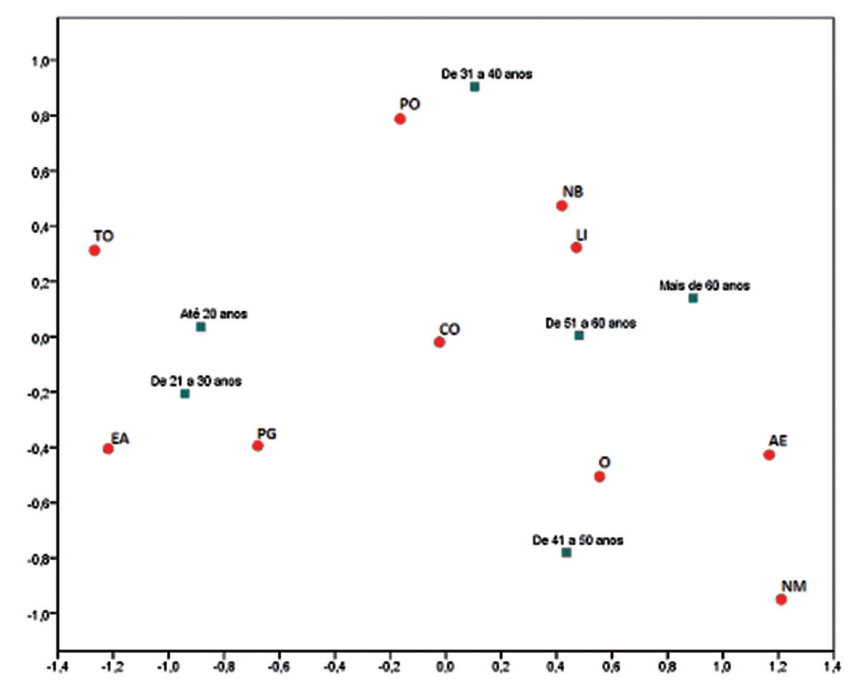

$E A=$ Estruturas anatômicas

$\mathrm{PG}=$ Patologia de glândula salivar

TO = Tumores odontogênicos

$\mathrm{PO}=$ Patologia óssea

$\mathrm{NB}=$ Neoplasia benigna

$\mathrm{NM}=$ Neoplasia maligna

$\mathrm{LI}=$ Lesões inflamatórias

$\mathrm{CO}=$ Cistos odontogênicos

$\mathrm{AE}=$ Alterações epiteliais

$\mathrm{O}=$ Outros

Figura 1 - Gráfico bidimensional da Anacor para sexo feminino

Com base nessa análise, é possível notar que existe uma relação íntima entre os pacientes homens de até 30 anos com os diagnósticos de estruturas anatômicas e patologias de glândulas salivares, assim como nos pacientes de 41 a 50 anos com as alterações epiteliais e nos de mais de 60 anos com as lesões inflamatórias e as neoplasias malignas. As mulheres têm relação semelhante quanto às lesões nos pacientes jovens, e com mais de 50 anos são mais frequentes as lesões inflamatórias e as neoplasias benignas.

\section{Discussão}

A maior incidência de diagnósticos histopatológicos foi o de folículo pericoronário, representando $15,4 \%$ do total de diagnósticos e, predominantemente, em pacientes até a terceira década de vida. Isso se explica pelo fato de o serviço ser referência em cirurgia bucomaxilofacial e pelo alto índice de procedimentos de remoção de dentes retidos aos quais há tecido aderido. A indicação de exame anatomopatológico é indicada sempre para assegurar que a lesão não constitui um processo patológico, visto a grande quantidade de lesões originárias de epitélio te. Uma Anacor resulta, entre outras informações, em um mapa bidimensional onde há tantos pontos quantas categorias de cada variável. A proximidade entre os pontos indica as associações entre eles. A Figura 1 ilustra essa associação por meio de mapa com o sexo feminino e a Figura 2, com o sexo masculino.

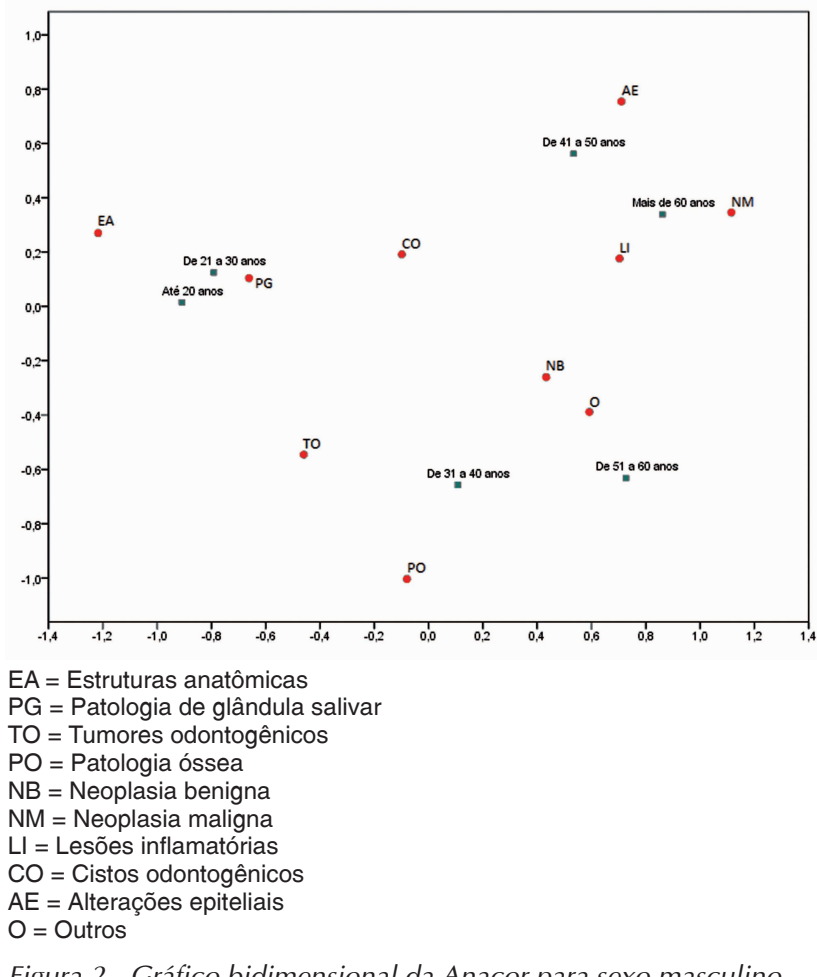

Figura 2 - Gráfico bidimensional da Anacor para sexo masculino

odontogênico ${ }^{13}$. A lesão foi enquadrada na categoria de estruturas anatômicas ( $\mathrm{N}=128$ e 18,1\% do total) e foi responsável por 109 diagnósticos, respondendo por $85 \%$ da categoria.

A hiperplasia papilar inflamatória, lesão com ocorrência maior em pacientes com mais de 40 anos de idade, responsável por 12,5\% do total dos diagnósticos, pode ser relacionada com pacientes usuários de próteses removíveis com muitos anos de uso, indo ao encontro dos estudos de Goiato et al. ${ }^{14}$ (2005) e de Maciel et al. ${ }^{15}$ (2008), justificada pela maior suscetibilidade da mucosa bucal ao trauma causado por essas próteses removíveis.

$\mathrm{Na}$ categoria dos tumores odontogênicos, responsável por $4,5 \%$ do total dos diagnósticos, foi incluído o diagnóstico de 11 lesões identificadas como ceratocisto, qualificado como tumor odontogênico ceratocístico de acordo com a classificação da Organização Mundial da Saúde (OMS) de 2004. O correto diagnóstico dos tumores odontogênicos é de fundamental importância para o cirurgião bucomaxilofacial, devido ao comportamento, ao tratamento e à morbidade variada de cada uma dessas patolo$\operatorname{gias}^{16,17}$.

As neoplasias malignas foram responsáveis por $5,1 \%$ dos casos, estes predominantemente em 
homens com mais de 60 anos de idade, resultado que vai de encontro com os valores encontrados nos estudos de Jones e Franklin² (2006) e de Mendez et al. $^{7}$ (2012). A lesão mais frequente foi a de carcinoma epidermoide, responsável por 58,3\% dos diagnósticos entre as neoplasias malignas, concordando com diversos estudos da literatura ${ }^{1-4,7,10-12}$.

A hiperparaceratose e a acantose, juntamente com a mucosa escamosa com acantose, foram os principais diagnósticos da categoria alterações epiteliais, que, somadas, são responsáveis por 90,3\% dos diagnósticos da categoria. O diagnóstico e o acompanhamento dessas lesões é de fundamental importância, por apresentarem sinais que podem ser considerados como cancerizáveis ${ }^{6,13,18}$.

Das lesões classificadas como neoplasias benignas, as mais frequentes foram de fibroma e papiloma, cada qual responsável por $22,6 \%$ da amostra, com 19 diagnósticos cada, concordando com os resultados dos estudos de Loureiro et al. ${ }^{4}(1997)$, Mendez et al. ${ }^{7}$ (2012) e Rocha et al. ${ }^{11}$ (2006).

Os cistos odontogênicos foram responsáveis por $13,6 \%(\mathrm{~N}=96)$ da amostra, e os diagnósticos mais comuns dessa categoria foram de cisto radicular (n $=63 / 65,6 \%)$ e cisto dentígero $(\mathrm{N}=24 / 25 \%)$, resultado semelhante ao dos estudos de Prockt et al. ${ }^{19}$ (2008) e Ochsenius et al. ${ }^{20}$ (2007).

Patologias classificadas como de glândulas salivares foram diagnosticadas 52 vezes $(7,4 \%)$, número de ocorrências que inclui apenas patologias associadas a glândulas salivares menores, porque o serviço não é referência para tratamento de patologias de glândulas salivares maiores, ficando a cargo do Serviço de Cirurgia de Cabeça e Pescoço. Laudos com o diagnóstico de sialoadenite, apesar de sua natureza inflamatória, foram incluídos nessa categoria.

Os ossos dos maxilares são acometidos por inúmeras patologias ósseas, e o seu diagnóstico é sempre um desafio para o cirurgião bucomaxilofacial. Com isso, o exame anatomopatológico torna-se um aliado indispensável ${ }^{2}$. No levantamento, foram classificados 71 diagnósticos como patologias ósseas, estas de 30 tipos diferentes. O diagnóstico de osteomielite foi classificado como patologia óssea, e não como lesão inflamatória.

Houve 26 diagnósticos $(3,7 \%)$ que não foram classificados em nenhuma outra categoria, tais como corpo estranho, candidíase, fibrose de fibras musculares, mucosa do trato respiratório, estes classificados como outros.

O Serviço de Cirurgia e Traumatologia Bucomaxilofacial no Complexo Hospitalar Santa Casa de Misericórdia de Porto Alegre atende a todos os hospitais do complexo e, assim, a pacientes de todas as faixas etárias, incluindo pacientes pediátricos oriundos do Hospital da Criança Santo Antônio.

Estudos têm demonstrado que outros fatores, além da idade e do sexo, como tabagismo, etilismo, uso de próteses removíveis e fatores socioeconômicos, podem estar associados à incidência de lesões do complexo bucomaxilofacial ${ }^{6,7,13}$. Infelizmente, esses dados, em geral, não são informados pelo cirurgião, nem repassados para fichas e laudos histopatológicos. Como essas informações quanto aos fatores comportamentais não estão disponíveis nos arquivos do laboratório, não foi possível avaliar esses fatores neste estudo.

\section{Conclusão}

O estudo concluiu que a maioria dos diagnósticos foi de lesões benignas, e as que tinham natureza inflamatória foram as mais recorrentes.

O folículo pericoronário foi a lesão mais frequente e fortemente associada com pacientes de até 30 anos, independentemente do sexo, assim como as patologias de glândulas salivares. As lesões inflamatórias estão mais presentes em mulheres com mais de 50 anos e em homens com mais de 60. As neoplasias malignas também estão mais presentes nos homens com mais de 60 anos de idade.

Fatores como idade e sexo podem ser auxiliares no diagnóstico diferencial para grande parte das lesões do complexo bucomaxilofacial.

\section{Abstract}

Ojective: this study assessed the prevalence of oral lesions by histopathological diagnosis, assisted by the service of maxillofacial surgery and traumatology at a hospital in southern Brazil, from the period of January 2000 to March 2012. Methods: data was obtained in the reports issued by the pathology laboratory. Seven hundred and seven histopathological reports were analyzed. Results: the results demonstrated that inflammatory lesions were the most frequent ones (21.4\%). Most often, the diagnosis affected women (60.3\%) and as for age group, patients up to 30 years old (40.4\%). The dental follicle (15.4\%) was the most frequent lesion and it was strongly associated with patients up to 30 years old regardless of gender, as well as diseases of the salivary glands. Conclusion: inflammatory lesions are more prevalent in women over age 50 and men over 60. Malignant neoplasms are also more prevalent in men over 60 years of age.

Keywords: Epidemiology. Diagnosis.

\section{Referências}

1. Leonel ECF, Vieira EH, Gabrielli MAC. Análise retrospectiva da incidência, diagnóstico e tratamento das lesões bucais encontradas no Serviço de Cirurgia e Traumatologia Bucomaxilofacial da Faculdade de Odontologia de Araraquara Unesp. Rev Paul Odontol 2002; 24(3):18-22.

2. Jones AV, Franklin CD. An analysis of oral and maxillofacial pathology found in adults over a 30-year period. J Oral Pathol Med 2006; 35(7):392-401. 
3. Moreira ARO, Oliveira CDM, Silva RR, Lopes FF, Bastos EG. Levantamento epidemiológico das doenças epiteliais da região bucomaxilofacial: casuística de 20 anos. Rev Gaúcha Odontol 2011; 59(1):65-70.

4. Loureiro MS, Duarte R, Figueiredo MAS, Lorandi CS, Yurgel LS. Levantamento epidemiológico dos diagnósticos histopatológicos de um laboratório de patologia bucomaxilofacial em um período de 18 anos. Rev Odonto Ciência 1997; 12(24):11730.

5. Lima GS, Fontes ST, Araújo LMA, Etges A, Tarquinio SBC, Gomes APN. A survey of oral and maxillofacial biopsies in children. A single-center retrospective study of 20 years in Pelotas-Brazil. J Appl Oral Sci 2008; 16(6):397-402.

6. Carrard VC, Haas AN, Rados PV, Filho MS, Opperman RV, Albandar JM, et al. Prevalence and risk indicators of oral mucosal lesions in an urban population from South Brazil. Oral Dis 2011; 17(2):171-9.

7. Mendez M, Carrard VC, Haas AN, Lauxen IS, Barbachan JJ, Sant'Ana Filho M, et al. A 10-year study of specimens submitted to oral pathology laboratory analysis: lesion occurrence and demographic features. Braz Oral Res 2012; 26(3):235-41.

8. Volkweis MR, Garcia R, Pacheco CA. Retrospective study of oral lesions in the population attended at the Dental Specialty Center. RGO - Rev Gaúcha Odontol 2010; 58(1):21-5.

9. Almeida JD, Cabral LAG. Diagnóstico do carcinoma bucal. RGO - Rev Gaúcha Odontol 1992; 40(3):167-70.

10. Borges FT, Garbin CAS, Carvalhosa AA, Casto PHS, Hidalgo LRC. Oral cancer epidemiology in a public laboratory in Mato Grosso State, Brazil. Cad Saúde Pública 2008; 24(9):1977-82.

11. Rocha DA, Oliveira LMM, Souza LB. Neoplasias Benignas da Cavidade Oral: Estudo Epidemiológico de 21 anos (1982 a 2002). Revista de Odontologia da Universidade Cidade de São Paulo 2006; 18(1):53-60.

12. Losi-Guembarovski R, Menezes RP, Poliseli F, Chaves VN, Kuasne H, Leichsenring A, et al. Oral Carcinoma Epidemiology in Paraná State, Southern Brazil. Cad Saúde Pública 2009; 25(2):393-400.

13. Neville BW, Day TA. Oral cancer and precancerous lesions. CA Cancer J Clin 2002; 52(4):195-215.

14. Goiato MC, Castelleoni L, Santos DM, Gennari Filho H, Assunção WG. Lesões orais provocadas pelo uso de próteses removíveis. Pesq Bras Odontoped Clin Integr 2005; 5(1):8590.

15. Maciel SV, Souza RSV, Donato LMA, Albuquerque IGM, Donato LFA. Prevalência das lesões de tecidos moles causadas por próteses removíveis nos pacientes da Faculdade de Odontologia de Caruaru, PE, Brasil. Pesq Bras Odontoped Clin Integr 2008; 8(1):93-7.

16. Araujo A, Gabrielli MFR, Medeiros PJ. Aspectos atuais da cirurgia e traumatologia bucomaxilofacial. 1. ed. São Paulo: Santos; 2007.

17. Fernandes AM, Duarte ECB, Pimenta FJGS, Souza LN, Santos VR, Mesquita RA, et al. Odontogenic tumors: a study of 340 cases in a Brazilian population. J Oral Pathol Med 2005; 34(10):583-7.
18. Rossi EP, Hirsch SA. A survey of 4,793 oral lesions with emphasis on neoplasia and premalignancy. J Am Dent Assoc 1977; 94(5):883-6.

19. Prockt AP, Schebela CR, Maito FD, Sant'Ana Filho M, Rados PV. Odontogenic cysts: analysis of 680 cases in Brazil. Head and Neck Pathology 2008; 29(4):150-6.

20. Ochsenius G, Escobar E, Godoy L, Penafiel C. Odontogenic cysts: analysis of 2,944 cases in Chile. Medicina Oral Patologia e Cirurgia Bucal 2007; 12(2):85-91.

Endereço para correspondência:

Bruno Trevisan

Rua Luiz Afonso, 395/ 203

Bairro Cidade Baixa

90050-310 Porto Alegre-RS

Fone: 32127243

E-mail: ctbmftrevisan@gmail.com

Recebido: 04/03/2013. Aceito: 11/05/2013. 